\title{
Relación entre salud y renuncia al empleo en trabajadoras de la industria maquiladora electrónica de Tijuana*
}

\author{
Sylvia Guendelman, Ph.D., ${ }^{(1)}$ Steven Samuels, Ph.D., ${ }^{(2)}$ Martha Ramírez-Zetina, M.C., M.S.P. ${ }^{(3)}$
}

\section{Guendelman S, Samuels S, Ramírez-Zetina M. Relación entre salud y renuncia al empleo en trabajadoras de la industria maquiladora electrónica de Tijuana. Salud Publica Mex 1999;41:286-296.}

\begin{abstract}
Resumen
Objetivo. Analizar factores de salud, laborales y sociales que contribuyen a renunciar al trabajo en dos maquiladoras transnacionales del ramo electrónico de Tijuana. Material y métodos Se realizó un estudio de cohorte a 725 mujeres empleadas en una planta japonesa y una estadunidense, entre enero de 1992 y marzo de 1994. La muestra se estratificó en dos intervalos de tiempo $\leq 30$ y $>30$ días laborados. Se efectuó un seguimiento de las mujeres hasta su renuncia o final del periodo de observación, mediante la recolección de información sobre variables de salud, sociales y ocupacionales de diversas fuentes de registro obtenidas de su centro de trabajo. Los motivos de renuncia y la confiabilidad de los datos obtenidos de los registros se estudiaron por medio de entrevistas de seguimiento realizadas a $46 \%(n=148)$ de las trabajado ras que renunciaron y fueron localizadas aproximadamente 12 meses (desviación estándar $=6.7$ ) después de su renuncia. Resultados La probabilidad acumulativa de renunciar al trabajo fue de $67 \%$ en el primer año y de $81 \%$ en el segundo. La ausencia de antecedentes laborales previos, turno diurno y nacionalidad de la compañía, resultaron factores predictivos para la renuncia al empleo durante los primeros 30 días. Tabaquismo, antecedentes quirúrgicos y haberse incapacitado por enfer medad general, después de controlar por otras variables, actuaron como factores predictivos para la renuncia posterior a
\end{abstract}

\author{
Guendelman S, Samuels S, Ramírez-Zetina M. \\ The relationship between health \\ and quitting work among electronic \\ industry female workers in Tijuana. \\ Salud Publica Mex 1999;41:286-296.
}

* Este estudio fue financiado por la Carnegie Corporation de N ueva York.

(1) Associate Professor, School of Public Health of California, Berkeley, Estados Unidos de América (EUA).

(2) Associate Adjunct Professor Maternal and Child Health Program, School of Public Health, University of C alifornia, Berkeley, EUA.

(3) Epidemiología, Unidad de Medicina Familiar N 0.19, Instituto Mexicano del Seguro Social (IMSS), Medicina Social Comunitaria,Tijuana, Baja California, México.

Fecha de recibido: 30 de julio de 1998 - Fecha de aprobado: 27 de enero de 1999

Solicitud de sobretiros: Sylvia Guendelman. Associate Professor, School of Public Health, University of California, Berkeley, California, United States of America.

Correo electrónico: sylviag@ uclink4.berkeley.edu 
30 días. En contraposición, la tasa de renuncia posterior a 30 días resultó menor para mujeres con enfermedades crónicas. Conclusiones. La renuncia al empleo en las maquiladoras es elevada y selectiva; en tanto que los factores ocupacionales se asociaron con la renuncia temprana, los relacionados con la salud fueron mejores factores predictivos para la renuncia posterior a los primeros 30 días.

Palabras clave: maquiladoras; salud de las mujeres; salud ocupacional; México kers quitting early, health factors are stronger predictors for quitting after 30 days.

Key words: electronic parts factories; women's health; occupational health; M exico
E n la frontera México-Estados Unidos de América (EUA), la mano de obra femenina es ampliamente utilizada por la industria maquiladora. A partir de la puesta en marcha del Tratado de Libre Comercio en 1994, el empleo en la industria maquiladora se incrementó en $20 \%$; las ramas electrónica y textil emplean el mayor número de mujeres ensambladoras de esta industria. ${ }^{1,2}$

Las maquiladoras han sido objeto de crítica atribuida a malas condiciones de trabajo, ${ }^{3,4}$ que incluyen $^{1}$ bajos salarios, operaciones repetitivas con ritmo acelerado, jornadas largas, insuficientes periodos de descanso, exposición a tóxicos químicos, mala ventilación, niveles de ruido excesivos y riesgo elevado de accidentes. ${ }^{3,5}$ Además, las trabajadoras denuncian la baja autonomía que poseen para tomar decisiones laborales, así como el escaso apoyo que reciben por parte de los supervisores. ${ }^{6}$

A pesar de las condiciones laborales adversas, existe escasa evidencia de que la salud de las ensambladoras experimente mayor deterioro en relación con la de las mujeres que laboran en sectores ajenos al maquilador, exceptuando por una disminución en el peso de sus recién nacidos. ${ }^{6,7}$ La identificación de los factores de salud, ocupacionales y sociales que propician que las mujeres abandonen el trabajo maquilador, podría contribuir a evaluar con mayor precisión si dicha ocupación resulta perjudicial para la salud.

La rotación de personal en las maquiladoras es elevada. En un informe ${ }^{2}$ reciente se ha estimado un promedio mensual de rotación en Tijuana de $12.7 \%$. Otras plantas a lo largo de la frontera informan ${ }^{3}$ rotaciones de 80 a $100 \%$ anual. ${ }^{8,9}$ Debido a que se sabe poco sobre el efecto del trabajo maquilador en la salud y el bienestar de las trabajadoras, resulta difícil definir si es la salud o son otros acontecimientos vitales los que influyen sobre la decisión de las mujeres para renunciar o dejar su empleo.

En investigaciones previas sobre mujeres que han renunciado al trabajo de maquila, se han citado ${ }^{1}$ como motivos de renuncia las obligaciones familiares, incluyendo el deseo de contraer matrimonio y el cuidado del hogar y los hijos. Otras razones argumentadas son bajos salarios, problemas de horario, necesidad de descanso tras un largo periodo de trabajo monótono y problemas con el supervisor. ${ }^{3}$ Sin embargo, no existe evidencia suficiente de que estos factores sean la causa exclusiva de las elevadas tasas de renuncia; ${ }^{8}$ de hecho, un factor importante podría ser la salud.

Las ensambladoras de la industria electrónica han informado acerca de irritación ocular, deterioro visual, dolores de cabeza y alergias, así como sintomatología emocional referida como nerviosismo, depresión y crisis de llanto. ${ }^{10}$

El propósito del presente estudio fue analizar los factores que contribuyen a la renuncia al empleo entre las trabajadoras de la industria maquiladora electrónica, y se realizó en torno a las siguientes interrogantes: ¿qué efecto tienen las condiciones laborales y las características sociodemográficas de las mujeres en la probabilidad de renunciar a la maquiladora?, ¿hasta qué punto los antecedentes de salud, y aquellos problemas de salud que ocurren durante el periodo de empleo en la planta, aumentan la probabilidad de renuncia?

\section{Material y métodos}

Se efectuó un estudio de cohorte con mujeres contratadas en dos plantas maquiladoras electrónicas de Tijuana, entre el 2 de enero de 1992 y al 31 de marzo de 1994. Se escogieron plantas electrónicas multinacionales debido a que el debate social respecto al impacto de las maquiladoras sobre la salud y bienestar del trabajador se ha centrado en estas empresas.

Se seleccionaron dos empresas, una japonesa que ensambla cámaras y equipos de video con aproximadamente 800 trabajadores, de los cuales $60 \%$ son mujeres, y otra estadunidense que ensambla equipo médico, incluyendo catéteres urinarios, circuitos respira- 
torios e instrumentos de diagnóstico con aproximadamente 650 trabajadores, de los cuales $58 \%$ son mujeres. Ambas operan cinco días a la semana, 24 horas al día, en tres turnos. Las trabajadoras recién contratadas reciben un entrenamiento mínimo. En comparación con la estadunidense, la planta japonesa tiene una jerarquía administrativa más rígida, reglas de ausentismo más estrictas y promueve notablemente los valores de lealtad laboral.

Los servicios de salud se ofrecen en ambas plantas. En la japonesa éstos operan 10 horas al día con dos niveles de atención: en el primero, una enfermera provee de cuidados básicos de salud y refiere los casos que ameritan al médico, quien constituye el segundo nivel de atención. Si la resolución del caso no es factible en la planta por su complejidad diagnóstica o terapéutica, o bien el trabajador requiere de incapacidad, el paciente es referido al Instituto Mexicano del Seguro Social (IMSS).

En la planta estadunidense, dos enfermeras y un médico proveen de atención a la salud en forma similar a la descrita para la planta japonesa. Ambos servicios de salud utilizan técnicas comparables de registro de actividades y archivo.

\section{Muestra}

Por medio de la gerencia de ambas maquiladoras, se obtuvieron listados de las trabajadoras que iniciaron labores entre el 2 de enero de 1992 y el 31 de marzo de 1994. Se identificaron las fechas de contratación y renuncia de cada trabajadora. Las participantes se limitaron a mujeres que trabajaron como operadoras de ensamblaje y cuya documentación personal y médica se encontraba en los archivos. De 928 mujeres, 203 (21.9\%) fueron eliminadas por carecer de examen médico de ingreso; la muestra final fue de 725 mujeres. Las tasas de renuncia fueron similares entre el grupo incluido y excluido del estudio. No se encontraron diferencias signficativas entre estos grupos respecto a la utilización de los servicios de salud de la planta o en el número, o tipo de incapacidades durante el periodo de empleo en la maquiladora. Sin embargo, más mujeres de la planta japonesa $(28.1 \%)$ que de la estadunidense (13.3\%) fueron excluidas por falta de registro de variables importantes $(p<0.05)$.

Debido a que en un estudio anterior se determinó que las probabilidades de renuncia al trabajo de ensamble y que las variables que afectan esa decisión varían según la duración en el empleo, ${ }^{11}$ la muestra se estratificó en dos periodos: de 1 a 30 días laborados y de 31 días o más.

\section{Variables y procedimientos de recolección de datos}

En cada planta, una asistente recabó datos de varias fuentes: el examen médico de ingreso, el registro diario médico y de enfermería, reportes de accidentes y control de incapacidades. Se utilizó el número de identificación de cada empleada para vincular los datos. Las asistentes recibieron entrenamiento durante una semana y tuvieron supervisión diaria para garantizar la uniformidad y la precisión en la recolección de datos. Como medida de control de calidad se validaron los datos en $10 \%$ de los casos. Sin embargo, fue imposible validar los datos procedentes de documentación administrativa proporcionada por las gerencias respectivas, tales como nómina y prestaciones de las empleadas. Las asistentes se mantuvieron ajenas a los objetivos del estudio y juraron confidencialidad para evitar que la información se filtrara a las líneas de producción.

El estudio recabó información sobre la salud de cada participante desde el día de la contratación hasta el día de la renuncia o final del periodo de observación (31 de marzo de 1994), con un máximo de 26 meses.

La variable dependiente fue la renuncia voluntaria o involuntaria al trabajo en la maquiladora. Las variables independientes incluyeron características sociodemográficas, laborales y de salud sustraídas del examen médico de ingreso. Las variables sociodemográficas fueron: edad, estado civil, escolaridad, estado de origen, y antecedentes laborales y de trabajo previo en maquiladora. Las variables laborales fueron turno de trabajo y nacionalidad de la empresa. En tanto que las variables de salud fueron antecedentes de tabaquismo y alcoholismo, antecedentes ginecobstétricos como gestaciones y ciclo menstrual, y antecedentes quirúrgicos, alérgicos y de enfermedades crónicas incluyendo hipertensión, cáncer y epilepsia.

Se hizo un seguimiento de la utilización que hacían las participantes de los servicios de salud de la planta, durante el tiempo de empleo en la maquiladora. Se estudiaron cuantitativa y cualitativamente todas las consultas efectuadas al personal médico y de enfermería de la planta, por demanda de servicios preventivos (exámenes de detección oportuna de enfermedades, planificación familiar o revisión médica); curativos (diagnóstico, tratamiento o referencia), y por causa de accidentes de trabajo.

Un médico especialista en medicina ocupacional y familiarizado con la industria electrónica seleccionó y clasificó, de acuerdo con la IX Revisión de la Clasificación Internacional de Enfermedades (CIE-9), ${ }_{12}^{2}$ las siguientes patologías en asociación ocupacional plausi- 
ble: dermatitis y enfermedades de la piel $(011,069)$; padecimientos de los ojos (036); conjuntivitis (037); padecimientos de los oídos (038); osteoartritis (071); padecimientos osteomusculares y del esqueleto $(072 \mathrm{y}$ 073), y lesiones (081-094). Excepto por las lesiones, los informes clínicos no fueron lo bastante detallados como para establecer una asociación causal entre las condiciones de salud mencionadas y el trabajo.

Para las mujeres que trabajaron más de 30 días se analizaron, además, las incapacidades por enfermedad general, maternidad y riesgos de trabajo, así como el número de días otorgados (con remuneración parcial o total) a consecuencia de éstas.

Los motivos de renuncia se obtuvieron de entrevistas de seguimiento a una muestra no representativa, que fueron $46 \%(n=148)$ de las trabajadoras que renunciaron y que fue posible localizarlas, aproximadamente 12 meses (desviación estándar-DE-=6.7) después de que habían dejado la planta. Esta submuestra resultó similar al resto de la población de estudio de trabajadoras que renunciaron, respecto a edad, escolaridad y antecedentes laborales. Se encontró una probabilidad más alta de que las participantes que renunciaron y fueron localizadas y sujetas a entrevista de seguimiento, fueran originarias de Baja California $(p=0.001)$.

\section{A nálisis de datos}

Se desarrolló un perfil de las trabajadoras según sus características sociodemográficas, laborales y de salud. El periodo de seguimiento se dividió en dos intervalos. El análisis del primer intervalo (renuncia a los 30 días o menos de empleo), se hizo por cruce de variables y regresión logística; el correspondiente al segundo intervalo (renuncia posterior a los 30 días de empleo) se realizó mediante tablas de vida y regresión múltiple. Para ajustar por diferencias de tiempo desde la contratación hasta la terminación del estudio, los análisis de tablas de vida estimaron la probabilidad de renunciar al trabajo por el número de días trabajados. Así, el dato considerado para las que renunciaron fue el número de días trabajados hasta la renuncia, mientras que para las mujeres que no renunciaron durante ese periodo, fue el número de días trabajados hasta el 31 de marzo de 1994 (observación "censurada"). Para el análisis mediante tablas de vida se dicotomizaron las variables independientes.

Para analizar los efectos simultáneos de las variables independientes en la probabilidad de renunciar al trabajo, se utilizó el modelo de Cox, conocido también como modelo de riesgos proporcionales. ${ }^{13}$ Primero se elaboró un modelo para observar si alguna característica sociodemográfica o de salud presente al momento del ingreso predecía la renuncia. En el segundo modelo, las características laborales del nuevo trabajo en la maquiladora, se añadieron al modelo de referencia. El tercer modelo añadió los eventos de salud ocurridos durante el empleo. La influencia de las variables independientes en un análisis de riesgos proporcionales, se mide por su efecto en la tasa semanal de renuncias, análogo a la tasa de mortalidad específica por edad. El efecto se resume por la tasa de riesgo (hazard ratio), que representa el efecto relativo en la tasa semanal de renuncias cuando se aumenta una variable independiente en una unidad. Una proporción de riesgo mayor de 1.0 indica que la variable está asociada con una renuncia más temprana. Esta aproximación analítica permitió dilucidar si los eventos de salud ocurridos durante este empleo eran mejores factores predictivos de renuncia que las características de salud que presentaba la mujer a su ingreso. Los cuadros descriptivos se elaboraron en los paquetes estadísticos SPSS y STATA 3.1, y las tablas de vida y análisis de regresión, en SAS y STATA.

\section{Resultados}

De las mujeres seleccionadas, 53\% trabajaban en la planta japonesa y $47 \%$ en la estadunidense. En ambas maquiladoras resultaron jóvenes ( 24 años, $\mathrm{DE}=6.4)$, predominantemente solteras $(55 \%)$ e inmigrantes foráneas de Baja California (79\%) (cuadro I). Aproximadamente la mitad había cursado únicamente educación primaria y carecía de experiencia previa de trabajo; $43 \%$ había trabajado anteriormente en maquiladora. La mayoría se desempeñaba en horario diurno (85\%) y sólo 2.3\% trabajaba en horario nocturno. La media trabajada fue de 84 días. Según los exámenes médicos de ingreso, $11.7 \%$ eran fumadoras y $11.6 \%$ consumían bebidas alcohólicas. Una proporción considerable declaró antecedentes quirúrgicos $(30.1 \%)$ y $6.2 \%$ antecedentes alérgicos. La prevalencia de enfermedades crónicas fue baja, de acuerdo con lo esperado para una población joven. De las trabajadoras, 78\% admitieron tener antecedentes de embarazo y, entre ellas, el promedio del número de gestaciones fue 1.7.

Para el final del periodo de observación, $65.5 \%$ de las mujeres habían recurrido a los servicios de la enfermera o del médico de la planta, por lo menos una vez. El promedio de atenciones de salud recibidas fue de 4.8 (DE= 8.2); de éstas, 4.5 fueron por servicios curativos. Las causas más frecuentes de atención médica fueron infecciones intestinales y respiratorias, dolores de cuello y cabeza, cólicos e irregularidades menstruales. De las trabajadoras, $46 \%$ requirieron atención 


\section{Cuadro I \\ Características de las trabajadoras de maquiladora estudiadas. Baja California, México, enero de 1992 a marzo de 1994}

\begin{tabular}{|c|c|c|}
\hline Total & $N=725$ & $\%$ \\
\hline \multicolumn{3}{|l|}{ A. Características de referencia } \\
\hline Edad, promedio en años (DE) & 719 & $23.5(6.4)$ \\
\hline \multicolumn{3}{|l|}{ Estado de orígen } \\
\hline Baja California & 154 & 21.3 \\
\hline 0 tro estado & 569 & 78.7 \\
\hline \multicolumn{3}{|l|}{ Estado Civil } \\
\hline Soltera & 397 & 54.8 \\
\hline Casada/unión libre & 296 & 41.2 \\
\hline Separada/divorciada & 29 & 4.0 \\
\hline \multicolumn{3}{|l|}{ Educación } \\
\hline Primaria & 378 & 53.2 \\
\hline Secundaria & 286 & 40.2 \\
\hline Preparatoria & 47 & 6.6 \\
\hline \multicolumn{3}{|l|}{ Trabajo previo } \\
\hline Sí & 377 & 52.0 \\
\hline No & 348 & 48.0 \\
\hline \multicolumn{3}{|l|}{ Trabajo previo en maquiladora } \\
\hline Sí & 311 & 42.9 \\
\hline No & 414 & 57.1 \\
\hline \multicolumn{3}{|l|}{ Compañía } \\
\hline Japonesa & 387 & 53.4 \\
\hline Estadunidense & 338 & 46.6 \\
\hline \multicolumn{3}{|l|}{ Turno } \\
\hline Día & 618 & 85.2 \\
\hline Tarde & 87 & 12.0 \\
\hline Noche & 17 & 2.3 \\
\hline Variable & 3 & 0.4 \\
\hline Días trabajados (mediana) & & 84 \\
\hline \multicolumn{3}{|l|}{ Prácticas de salud } \\
\hline Fuma actualmente & 85 & 11.7 \\
\hline Bebe actualmente & 84 & 11.6 \\
\hline \multicolumn{3}{|l|}{ Historial médico } \\
\hline Cirugía & 218 & 30.1 \\
\hline Alergias & 45 & 6.2 \\
\hline \multicolumn{3}{|l|}{ Enfermedades crónicas: } \\
\hline Hipertensión & 25 & 3.5 \\
\hline Cáncer & 8 & 1.1 \\
\hline Epilepsia & 2 & 0.3 \\
\hline Ciclos menstruales irregulares & 36 & 5.0 \\
\hline Embarazos, número promedio de, (DE) & 559 & $1.7(1.8)$ \\
\hline \multicolumn{3}{|l|}{ B. Información de seguimiento } \\
\hline \multicolumn{3}{|l|}{ Utilización de servicios de salud } \\
\hline Al menos una consulta médica o de enfermería & 465 & 65.5 \\
\hline Promedio de consultas (DE) & 725 & $4.8(8.2)$ \\
\hline Al menos una consulta ocupacional & & 457. \\
\hline $\begin{array}{l}\text { medica o de enfermeria } \\
\text { Promedio de consultas (DE) }\end{array}$ & $\begin{array}{l}331 \\
725\end{array}$ & $\begin{array}{c}45.1 \\
1.4(2.8)\end{array}$ \\
\hline \multicolumn{3}{|l|}{ Incapacidades debido a: } \\
\hline Enfermedad general & 59 & 9.9 \\
\hline Maternidad & 22 & 3.7 \\
\hline Riesgos de trabajo & 12 & 2.0 \\
\hline \multicolumn{3}{|l|}{$\begin{array}{l}\text { Promedio de días de incapacidad/persona } \\
\text { según tipo de incapacidad: }\end{array}$} \\
\hline Enfermedad general, promedio (DE) & 59 & $12.3(18.3)$ \\
\hline Maternidad, promedio (DE) & 22 & $62.9(25.8)$ \\
\hline Riesgos de trabajo, promedio (DE) & 12 & $28.7(46.3)$ \\
\hline
\end{tabular}

por lo menos una vez por padecimientos relacionados con salud ocupacional, principalmente por sintomatología de cuello y cabeza, dolores osteomusculares y lesiones. No obstante, no se puede afirmar que las atenciones clasificadas como ocupacionales se debieran a factores ocupacionales o que las clasificadas como no ocupacionales no hayan tenido orígenes de índole ocupacional.

De acuerdo con lo informado por las gerencias, 9.9\% de las mujeres que trabajaron más de 30 días recibieron incapacidad por enfermedad general. Estas mujeres no presentaban más antecedentes de padecimientos crónicos respecto a las que no se incapacitaron; $3.7 \%$ recibieron incapacidad por maternidad y $2 \%$ por riesgo de trabajo. El número promedio de días de incapacidad por enfermedad general fue de $12.3(\mathrm{DE}=$ $18.3)$; por maternidad, de $62.9(\mathrm{DE}=25.7)$, y por accidentes, de $28.7(\mathrm{DE}=46.3)$.

Las estimaciones realizadas con el método KaplanMeir indicaron que en los primeros 30 días, 121 (16.7\%) de las 725 empleadas renunciaron. Como se muestra en el cuadro II, las mujeres sin experiencia laboral previa tuvieron una probabilidad dos veces mayor de renunciar en los primeros 30 días que aquellas con antecedentes laborales $(p=0.0001)$. Las ensambladoras de la planta japonesa tuvieron una probabilidadad ocho veces mayor de renunciar que las ensambladoras de la planta estadunidense ( $p=0.0001)$, y las que trabajaban en turno diurno tuvieron más de tres veces la probabilidad de renunciar en los primeros 30 días que las que trabajaban otro turno $(p=0.001)$. Las condiciones de salud al ingreso no incrementaron significativamente el riesgo de renuncia temprana.

Al calcularse la probabilidad de renuncia previa a 30 días, utilizando modelos de regresión logística que incluían todas las variables indicadas en el cuadro II, excepto nacionalidad de la compañía, los únicos factores predictivos que resultaron significativos fueron: ausencia de experiencia laboral y turno diurno (cuadro III). Cuando se añadió la nacionalidad de la compañía al modelo de regresión logística, este factor resultó significativo (RM 8.4, IC 95\% 4.2-16.5) y los efectos de experiencia laboral previa y turno, disminuyeron y no fueron significativos. Al agregarse al modelo de utilización de servicios de salud durante el empleo, las variables no incidieron significativamente en el riesgo de renuncia temprana.

Para las mujeres que trabajaron más de 30 días, el análisis mediante tablas de vida estimó la probabilidad de renuncia desde el momento de contratación, controlando por el número de días trabajados. Estos análisis permitieron predecir que $64 \%$ de las trabajadoras habrían dejado el trabajo en 365 días y $79 \%$ 


\section{Cuadro II \\ Probabilidad de renuncia al trabajo EN LOS PRIMEROS 30 DÍAS DE EMPLEO POR Características de referencia. Baja California, MéXico, enero de 1992 a marzo de 1994}

\begin{tabular}{l} 
Total \\
Características sociodemográficas \\
Edad \\
$\quad<22$ Años \\
\hline 22 Años
\end{tabular}

$N=12$

$\%$ de riesgo

p

Estado de origen

Baja California

0 tro estado

(53)

(68)

$\begin{array}{ll}16.4 & 0.76 \\ 17.2\end{array}$

\begin{tabular}{llll} 
Estado Civil & & & \\
No casada & $(67)$ & 15.7 & 0.41 \\
\hline Casada & $(54)$ & 18.1 &
\end{tabular}

Historia laboral previa No

22.8

$2.8 \quad 0.0001$

\begin{tabular}{lcrc}
$\begin{array}{l}\text { Educación } \\
\text { Primaria }\end{array}$ & $(69)$ & 18.4 & 0.29 \\
\hline Secundaria 0 superior & $(51)$ & 15.5 & \\
$\begin{array}{l}\text { Características laborales } \\
\text { Compañía } \\
\quad \text { Estadunidense }\end{array}$ & & & \\
\hline Japonesa & $(13)$ & 3.3 & 0.0001 \\
\hline & $(108)$ & 27.5 &
\end{tabular}

\begin{tabular}{lrrr}
$\begin{array}{l}\text { Turno de trabajo } \\
\text { Día }\end{array}$ & (115) & 18.6 & 0.001 \\
\hline No día & $(6)$ & 5.6 &
\end{tabular}

\section{Características de salud} Fumadora

\begin{tabular}{cccc} 
No & $(102)$ & 16.1 & 0.13 \\
\hline Sí & $(19)$ & 22.6 &
\end{tabular}

Antecedentes quirúrgicos

\begin{tabular}{llll} 
No 0 & $(82)$ & 16.2 & 0.57 \\
\hline Sí & $(39)$ & 17.9 &
\end{tabular}

Historial de enfermedades crónicas*

\begin{tabular}{cccc} 
No & $(111)$ & 17.1 & 0.36 \\
\hline Sí & $(10)$ & 13.5 &
\end{tabular}

* Incluyendo alergias

habrían renunciado en 730 días (cuadro IV). La estratificación por compañía mostró que las trabajadoras de la planta japonesa tenían una probabilidad levemente mayor de renunciar que las trabajadoras de la estadunidense ( $71 \%$ vs. $60 \%$ a los 365 días). Entre las mujeres que trabajaron más de 30 días, el periodo de empleo previo a la renuncia varió significativamente de acuerdo con las características laborales y de salud presentes a su ingreso, pero no así con las características sociodemográficas. Esto se evaluó con la prueba log-rank, comparando las tasas semanales de renuncia entre dos grupos. Las empleadas que no desempeñaban trabajo diurno $(p=0.06)$ tuvieron porcentajes de renuncia más altos que las que trabajaban durante el día. Mujeres fumadoras $(p=0.008)$ y con antecedentes quirúrgi$\cos (p=0.004)$ renunciaron en mayor proporción que las no fumadoras y sin historial quirúrgico. En contraposición, las mujeres con antecedentes de enfermedades crónicas y alergias al ingreso registraron porcentajes de renuncia inferiores a las mujeres que negaron ser portadoras de enfermedades crónicas $(p=0.02)$. De manera similar, las mujeres que se incapacitaron por enfermedad general durante el periodo de empleo mostraron porcentajes de renuncia mayores en relación con las mujeres que no registraron incapacidades $(p=0.0001)$.

Las características laborales y de salud que resultaron estadísticamente significativas para la renuncia posterior a 30 días laborados y que se muestran en el cuadro IV, fueron incorporadas sucesivamente en tres modelos de regresión. El primer modelo examinó el efecto de los antecedentes laborales previos y las condiciones de salud al ingreso (tabaquismo, antecedentes quirúrgicos y enfermedades crónicas) sobre la probabilidad de renuncia. El segundo modelo añadió las características laborales del presente empleo (compañía solamente, pues estaba correlacionada con turno). Como se muestra en el cuadro $V$, después de controlar por estos factores, la probabilidad de renunciar al trabajo resultó 1.5 veces superior entre las fumadoras, 1.4 veces mayor entre las mujeres con historial quirúrgico y 1.2 veces más alto en trabajadoras de la planta japonesa. En cambio, las mujeres con historial de enfermedades crónicas tuvieron una probabilidad 36\% menor de renuncia posterior a los 30 días laborados en comparación con las mujeres que no refirieron enfermedades crónicas a su ingreso.

Estos cuatro factores predictivos fueron agregados a un tercer modelo de regresión que incluía los eventos de salud ocurridos durante el periodo de empleo, es decir, consultas médicas o de enfermería en la planta por motivos preventivos, curativos o de accidente laboral, así como incapacidades por enfermedad general, maternidad o riesgos de trabajo. En el análisis se consideraron el tipo de consultas y el número total. De los eventos de salud ocurridos durante el periodo de empleo, dos de ellos pronosticaron la renuncia al 


\section{Cuadro III \\ Factores predictivos de renuncia al trabajo previa a 30 días según características sociodemográficas, laborales y de salud (resultados del modelo de regresión logística). Baja California, México, ENERO DE 1992 A MARZO DE 1994}

\begin{tabular}{|c|c|c|c|c|}
\hline Variables & Razón de momios & $\mathrm{IC} 95 \%$ & Razón de momios & IC95\% \\
\hline Turno de trabajo diurno vs. otro & 3.0 & $1.24-7.27$ & 1.4 & $0.53-3.57$ \\
\hline Con historia laboral vs. sin & 2.5 & $1.58-3.85$ & 1.1 & $0.67-1.82$ \\
\hline Baja California vs. otro estado & 0.90 & $0.54-1.49$ & 1.0 & $0.62-1.78$ \\
\hline Estado civil casada vs. no casada & 1.2 & $0.80-1.92$ & 1.2 & $0.79-1.95$ \\
\hline Edad & 0.97 & $0.93-1.0$ & 0.97 & 0.93-1.01 \\
\hline Fumadora vs. no fumadora & 1.7 & $0.92-3.06$ & 1.5 & $0.81-2.84$ \\
\hline Con antecedentes quirúrgicos vs. sin & 1.3 & $0.79-2.01$ & 1.2 & $0.73-1.98$ \\
\hline Historial de enfermedades crónicas vs. sin & 0.8 & $0.39-1.64$ & 1.2 & $0.56-2.58$ \\
\hline Educación primaria vs. secundaria & 1.2 & $0.78-1.78$ & 1.1 & $0.70-1.66$ \\
\hline Planta japonesa vs. estadunidense & & & 8.4 & $4.23-16.5$ \\
\hline
\end{tabular}

trabajo posterior a 30 días laborados (cuadro V). Las mujeres que se incapacitaron por enfermedad general tuvieron una probabilidad 1.4 veces más alta de renunciar cada semana después de regresar al trabajo en comparación con las que no registraron ninguna incapacidad. Aunque el intervalo de confianza en el caso de incapacidad no sobrepasaba 1.0, la ausencia de significancia estadística al nivel $p=0.05$ pudiera atribuirse al pequeño número de mujeres con incapacidad. El tabaquismo, y los antecedentes quirúrgicos y de enfermedades crónicas continuaron siendo importantes factores predictivos en el modelo, mientras que el efecto de la nacionalidad de la compañía resultó ser menos relevante.

Las entrevistas de seguimiento a 148 mujeres en la comunidad confirmaron algunos de los hallazgos descritos en el cuadro V. Hubo más mujeres que declararon haber dejado su trabajo por motivos personales o familiares (56\%) que por aquellos relacionados con el trabajo o con la escuela (37\%), o por emigrar a EUA $(6.8 \%)$. Entre los motivos familiares más comunes se encontraron: embarazo, matrimonio, enfermedades generales no ocupacionales, obligaciones familiares como cuidado de los hijos, o dejar la ciudad para atender a un familiar enfermo, y la oposición del esposo o compañero al trabajo de la mujer.

\section{Discusión}

Esta investigación es una de las primeras en tener acceso directo a los datos generados por la propia industria maquiladora. En concordancia con los hallazgos de investigaciones previas, se encontró un alto porcentaje de rotación entre el personal de ensamblaje: casi
$17 \%$ de las operarias renunciaron en los primeros 30 días de empleo. Al finalizar los 26 meses del estudio, $54 \%$ de las trabajadoras habían renunciado. La probabilidad de renuncia, voluntaria o involuntaria, al trabajo en las plantas maquiladoras en función del número de días trabajados fue de $67 \%$ en un año y de $81 \%$ en dos años de empleo.

Las características laborales, como turno de trabajo y nacionalidad de la compañía, resultaron determinantes para la renuncia durante los primeros 30 días. La mayor rotación en la planta japonesa comparada con la estadunidenses podría atribuirse a reglamentos laborales más estrictos, controles mas rigurosos respecto a las incapacidades, o bien, a la reubicación geográfica de la planta japonesa sucedida justo antes del inicio del estudio. Las prácticas gerenciales podrían haberse visto afectadas por la reorganización y expansión de la planta. Salvo la ausencia de historial laboral previo, no se encontraron otras características sociodemográficas o condiciones de salud que predijeran la renuncia temprana al trabajo, incluso después de controlar por otros factores.

Las características sociodemográficas de las mujeres que trabajaron por más de 30 días tampoco contribuyeron significativamente a la probabilidad de renuncia. Sin embargo, algunas condiciones y eventos de salud ocurridos durante el empleo, sí contribuyeron; al controlar por factores laborales se encontró que la probabilidad de renuncia aumentó en las trabajadoras fumadoras o con antecedentes quirúrgicos y en aquellas que durante su empleo se incapacitaron por enfermedad general o maternidad. Debido a que casi $30 \%$ de las mujeres que trabajaron por lo menos 31 días contaban con antecedentes quirúrgicos, $10 \%$ eran 


\section{Cuadro IV}

Proporción de trabajadoras empleadas por más de 30 días que renuncian al trabajo

A LoS 90, 180, 365, 455, Y 730 DÍAS POR CARACTERÍSTICAS SOCIODEMOGRÁFICAS, LABORALES Y DE SALUD: estimaciones según tablas de vida. Baja California, México, enero de 1992 a marzo de 1994

\begin{tabular}{|c|c|c|c|c|c|c|}
\hline \multirow[b]{2}{*}{ Características } & \multicolumn{6}{|c|}{$\%$ que renuncia en los siguientes días } \\
\hline & $\mathrm{N}$ & $\begin{array}{c}90 \\
\%(S . E .)\end{array}$ & $\begin{array}{c}180 \\
\%(S . E .)\end{array}$ & $\begin{array}{c}365 \\
\% \text { (S.E.) }\end{array}$ & $\begin{array}{c}455 \\
\%(S . E .)\end{array}$ & $\begin{array}{c}730 \\
\%(S . E .)\end{array}$ \\
\hline Total & 598 & $28(02)$ & $43(02)$ & $64(02)$ & $70(03)$ & $79(03)$ \\
\hline
\end{tabular}

Sociodemográficas: Edad

\begin{tabular}{llllllll}
$\leq 22$ Años & 304 & $28(03)$ & $44(03)$ & $67(04)$ & $73(04)$ & $81(04)$ & 0.61 \\
\hline$>22$ Años & 294 & $28(03)$ & $42(03)$ & $61(03)$ & $67(04)$ & $77(05)$ &
\end{tabular}

Estado de origen

\begin{tabular}{llllllll} 
Baja California & 127 & $27(02)$ & $42(02)$ & $63(03)$ & $69(03)$ & $77(03)$ & 0.26 \\
\hline O tro estado & 470 & $29(03)$ & $47(05)$ & $68(05)$ & $77(05)$ & $87(05)$ & \\
Estado civil & & & & & & & \\
No casada & 330 & $24(02)$ & $41(03)$ & $66(03)$ & $71(04)$ & $80(04)$ & 0.55 \\
\hline Casada & 268 & $32(03)$ & $46(03)$ & $61(03)$ & $70(04)$ & $78(04)$
\end{tabular}

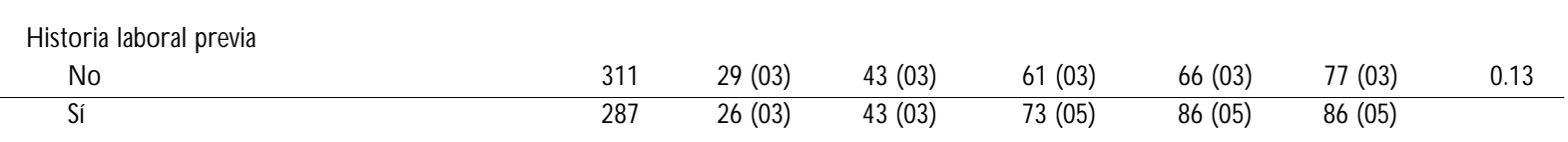

\begin{tabular}{llllllll}
$\begin{array}{l}\text { Educación } \\
\text { Secundaria o superior }\end{array}$ & 279 & $25(03)$ & $42(03)$ & $67(040$ & $72(04)$ & $80(04)$ & 0.93 \\
\hline Primaria & 305 & $30(03)$ & $45(03)$ & $60(03)$ & $75(04)$ & $75(04)$
\end{tabular}

\begin{tabular}{|c|c|c|c|c|c|c|c|}
\hline \multicolumn{8}{|l|}{ Laboral } \\
\hline \multicolumn{8}{|l|}{ Compañia } \\
\hline Americana & 274 & $29(03)$ & $40(03)$ & $60(03)$ & $66(03)$ & $76(03)$ & 0.06 \\
\hline Japonesa & 324 & $26(03)$ & $47(03)$ & $71(04)$ & $71(04)$ & $78(05)$ & \\
\hline \multicolumn{8}{|l|}{ Turno de trabajo } \\
\hline No turno diurno & 118 & $37(05)$ & $49(05)$ & $70(05)$ & $74(05)$ & $84(05)$ & 0.06 \\
\hline Turno diurno & 480 & $26(02)$ & $42(02)$ & $63(03)$ & $70(03)$ & $78(04)$ & \\
\hline
\end{tabular}

Salud

\begin{tabular}{lrrrrrrr}
$\begin{array}{l}\text { Fumadora } \\
\text { No }\end{array}$ & 533 & $25(02)$ & $42(02)$ & $63(03)$ & $70(03)$ & $79(03)$ & 0.008 \\
\hline Sí & 65 & $46(07)$ & $56(07)$ & $72(07)$ & $76(07)$ & $89(07)$ &
\end{tabular}

\begin{tabular}{l}
$\begin{array}{l}\text { Antecedentes quirúrgicos } \\
\text { No }\end{array}$ \\
\hline Sí
\end{tabular}

Historial de enfermedades crónicas y alergias
No
Sí

\begin{tabular}{|c|c|c|c|c|c|c|c|}
\hline No tuvo días de incapacidad & 513 & $30(02)$ & $48(02)$ & $67(03)$ & $72(03)$ & $84(03)$ & 0.0001 \\
\hline Tuvo al menos un día de incapacidad & 85 & $7(03)$ & $15(04)$ & $46(06)$ & $58(07)$ & $58(07)$ & \\
\hline
\end{tabular}




\section{Cuadro V \\ Factores predictivos de renuncia al empleo a más de 30 días (modelo de regresión multivariada de Cox). Baja California, México, enero de 1992 a marzo de 1994}

\begin{tabular}{|c|c|c|c|c|c|c|c|}
\hline \multirow[b]{2}{*}{ Variables } & \multirow[b]{2}{*}{ Prevalencia \% } & \multicolumn{3}{|c|}{ Modelo 1-2 } & \multicolumn{3}{|c|}{ Modelo 3} \\
\hline & & Tasa de riesgo & $p$ & IC95\% & Tasa de riesgo & $p$ & IC95\% \\
\hline \multicolumn{8}{|l|}{ Antecedentes de salud } \\
\hline Fumadora vs. no fumadora & 10.9 & 1.5 & 0.02 & $1.06-2.04$ & 1.4 & 0.03 & $1.03-2.00$ \\
\hline Con antecedentes quirúrgicos vs. sin & 29.3 & 1.4 & 0.008 & $1.08-1.74$ & 1.4 & 0.008 & $1.08-1.73$ \\
\hline Historial de enfermedades crónicas vs. sin & 5.0 & 0.64 & 0.02 & $0.44-0.94$ & 0.62 & 0.02 & $0.42-0.92$ \\
\hline \multicolumn{8}{|l|}{ Laboral } \\
\hline Planta japonesa vs. estadunidense & - & 1.2 & 0.11 & $0.95-1.51$ & 1.2 & 0.10 & $0.96-1.52$ \\
\hline \multicolumn{8}{|l|}{ Eventos de salud durante el empleo } \\
\hline Incapacidad por enfermedad general & $9.9 *$ & & & & 1.4 & 0.08 & $0.96-2.14$ \\
\hline Incapacidad por maternidad & $3.7^{*}$ & & & & 1.4 & 0.40 & $0.63-3.13$ \\
\hline
\end{tabular}

fumadoras y $10 \%$ más experimentaron alguna enfermedad o condición que requirió de incapacidad médica, se concluyó que los eventos de salud contribuyen significativamente a la rotación de personal en las maquiladoras. El tabaquismo en la mujer trabajadora de la industria maquiladora podría ser un indicador de mayor autonomía que refleja mayor poder de decisión y que le permite cambiar de trabajo más a menudo que otras mujeres. Es necesario llevar a cabo más estudios a fin de comprender la contribución del tabaquismo en la decisión de las mujeres a renunciar a su empleo.

Por otra parte, no todos los eventos de salud aumentan necesariamente la probabilidad de renuncia al trabajo. De hecho, el antecedente de alguna enfermedad crónica se comportó como factor disuasivo. Las mujeres con enfermedades crónicas no mostraron más predisposición a incapacitarse por enfermedad general que el resto de las mujeres. Quizá las enfermedades crónicas disminuyan la probabilidad de renuncia al trabajo porque las trabajadoras saben que otras maquiladoras investigan la existencia de condiciones crónicas antes de controlarlas y temen ser rechazadas. Otro motivo podría ser que su renuncia implica perder la cobertura médica y las prestaciones que otorga el IMSS.

En forma contraria a lo que se esperaba, la nacionalidad de la compañía no resultó ser determinante para la renuncia al trabajo tras los primeros 30 días de empleo, después de controlar por factores de salud. El hecho de que la planta japonesa haya mostrado probabilidades ligeramente más altas de renuncia en comparación con la estadunidense puede adjudicarse, también en este caso, a los motivos ya mencionados sobre reglamentación estricta y una reubicación geográfica que la trasladó hacia un sector menos accesible de la ciudad, justo antes de iniciarse este estudio.

Además de los eventos de salud, las obligaciones familiares comúnmente relacionadas con las razones de salud mental, parecen contribuir a la decisión de dejar la maquiladora. Las mujeres tienen la doble responsabilidad de aportar un ingreso económico y de atender a su familia. Si bien el diseño del estudio no incluyó mediciones directas para evaluar el efecto específico de las obligaciones familiares en la probabilidad de renuncia, las entrevistas de seguimiento realizadas a un grupo no representativo de mujeres, aproximadamente 12 meses después de que habían dejado la planta, revelaron que las obligaciones familiares desempeñan un papel importante en sus decisiones. Más de la mitad de las mujeres entrevistadas manifestaron que el cuidado de la familia, el matrimonio, o bien, la oposición de la pareja al trabajo de la mujer contribuyeron, al menos temporalmente, a su renuncia.

Otra consideración en el diseño del estudio que merece mención, es que los efectos de la salud en la probabilidad de renuncia pueden haberse atenuado debido a que los servicios médicos en las empresas intervinieron previniendo o tratando condiciones que de otra manera habrían conducido a la renuncia. En estudios previos se ha encontrado que la presencia de un médico o enfermera en la maquiladora disminuye la probabilidad de enfermedades o lesiones relacionadas con el trabajo. ${ }^{14}$ Por otra parte, los médicos de las plantas maquiladoras generalmente no están ca- 
pacitados en salud ocupacional, lo cual puede ocasionar que no correlacionen problemas de salud ocupacional con efectos del trabajo. Escasas trabajadoras $(1.7 \%)$ fueron referidas al IMSS para valoraciones más especializadas o incapacidad, si bien ellas tienen la opción de acceso directo a esa istitución de seguridad social.

A pesar de que los servicios médicos y de enfermería ofrecían atención primaria a la salud y no se limitaban a tratar exclusivamente afecciones ocupacionales o accidentes de trabajo, es posible que algunas ensambladoras hayan recibido atención fuera de las plantas y que esos eventos no estuvieron registrados en los expedientes. Este estudio carece de información sobre consultas de salud realizadas fuera de las plantas. Asimismo cabe la posibilidad de que las trabajadoras con el fin de obtener el empleo, no hayan reportado todos sus problemas de salud.

Los hallazgos de este estudio deben interpretarse con precaución, puesto que se limitan a dos plantas transnacionales que ofrecen servicios de salud, y por tanto no pueden generalizarse a plantas más pequeñas que no brindan estos servicios. Además, ambas maquiladoras ofrecen otros beneficios como cafeterías subvencionadas, cupones de comida y pequeñas subvenciones para el transporte que pueden reducir los efectos de los eventos de la salud en la rotación. Los hallazgos también se limitan a la calidad de la información registrada rutinariamente en las plantas. Algunas variables importantes como las relaciones entre supervisor y trabajadora y entre los mismos trabajadores, incluyendo acoso sexual y conflictos laborales, no estuvieron disponibles. Es importante destacar la necesidad de llevar a cabo otros estudios para determinar si los resultados de éste, pueden aplicarse a otras plantas con diferentes servicios médicos, estilos de gerencia y riesgos para la salud relacionados con exposición a otros agentes químicos o factores ergonómicos. ${ }^{15,16}$ Variables como la ubicación geográfica y las relacionadas con prestaciones, como subvención de vivienda o transporte, guarderías infantiles y cupones para alimentos, también pueden afectar la probabilidad de renuncia y ameritan ser estudiadas.

A pesar de las limitaciones mencionadas, la información que se obtuvo permite concluir que, al menos en estas dos plantas, los factores de salud inciden en la decisión de las mujeres para dejar la maquiladora. Si bien fumar podría ser un indicador de mayor autonomía que refleja acciones de emancipación de la mujer trabajadora, se trata de un hábito nocivo para la salud. Un paso importante en la promoción de un ambiente saludable de trabajo, es dirigir esfuerzos al grupo de mujeres fumadoras y a las que inciden en incapacida- des. Resultaría beneficioso proveer de un servicio de consejeras o trabajadoras sociales que promuevan programas que, por un lado, refuercen actitudes y comportamientos de mayor autonomía y autosuficiencia en la mujer, pero que, a su vez desplacen el hábito de fumar hacia acciones más saludables. Asimismo, sería recomendable que brinden asesoría y promuevan la formación de grupos de apoyo para las mujeres enfermas, además de terapia familiar y solución de conflictos con una red de referencia a organizaciones más especializadas para los casos que lo necesiten. Las anteriores medidas podrían llevar a reducir el porcentaje de renuncia, al tiempo que se mejoran las condiciones de vida de las trabajadoras.

Las investigaciones que se realicen posteriormente respecto a las mujeres trabajadoras en la industria maquiladora deberán considerar la influencia de los factores de salud en la selección y rotación del personal empleado en las líneas de ensamblaje de esa industria. De acuerdo con los hallazgos de este estudio, no son sólo los administradores quienes seleccionan trabajadoras saludables y jóvenes, sino que con frecuencia las trabajadoras mismas dejan la maquiladora debido a eventos relacionados con su estado de salud.

\section{Agradecimientos}

Se agradece al equipo de Medicina Social Comunitaria de Tijuana por su apoyo, a los doctores Mónica Jasis, por sus ideas en la etapa inicial del diseño de este estudio, Mark Hudes, por su apoyo en el análisis de datos, y Stephen McCurdy, por identificar los padecimientos de salud con posibles causas ocupacionales. Asimismo, se agradecen las valiosas observaciones que hicieron los dos revisores asignados por Salud Pública de México, y cuya labor contribuyó a mejorar la calidad de este artículo.

\section{Referencias}

1.C arrillo J.Transformaciones en la industria maquiladora de exportación. En: González-A réchiga B, Barajas-Escamilla R, eds. Las maquiladoras: ajuste estructural y desarrollo regional.Tijuana: El Colegio de la Frontera del Norte, 1989:37-54.

2. Pedrero N ieto M.The economically active population in the N orthern region of Mexico. En:W eeks R, Ham-Chande R, eds. Demographic dynamics of the U.S.-Mexico border. El Paso,Texas:W estern Press, 1992.

3. Fernández-Kelly M. For we are sold, I and my people. Albany, N ueva York: State University of N ew York Press, 1983.

4. Fuentes A, Ehrenreich B. Women in the global factory. Boston: South End Press, 1983. 
5. Hovell M, Sipan C, Hofstetter C, D uBois BC, Krefft A, Conway J et al. $O$ ccupational health risks for Mexican women:The case of the maquiladora along the Mexican-United States border. Int J Health Serv 1988;18: 617-627.

6. G uendelman $S$, Jasis $M$. The health consequences of maquiladora work: Women on the U.S.-Mexican border. Am J Public Health 1993;83:37-44.

7. Eskenazi B, Guendelman S, Elkin E, Jasis M. Preliminary study of the reproductive outcomes of maquiladora workers in Tijuana, Mexico. Am J Ind Med 1993;24:667-676.

8. Sklair L.Assembling for development:The maquila industry and the United States. Boston: Unwin Hyman Press, 1989.

9. Stoddard E. Maquila:Assembly plants in N orthern Mexico. El Paso,Texas: Texas W estern Press, 1987.

10. Guendelman S, Jasis M. Electronics and garment maquiladoras in Tijuana:The health of working women. Border Health 1992;8:41-55.

11. G uendelman S, Samuels S, Ramírez M. Women who quit maquiladora work on the U.S.-Mexico border:Assessing health, occupation and social dimensions in two transnational electronic plants. Am J Ind Med 1998; 33:501-509.
12. U.S. D epartment of Health and Human Services. ICD-9-CM, International Classification of D iseases, 9th Revision. 3a. edición. 0 ctubre, 1988; vol. 3.

13. Cox D. Regression models and life tables (with discussions). J Royal Stat Soc, Series B, 1972;30:248-275.

14. Balcázar H, Denman C, Lara F. Factors associated with work-related accidents and sickness among maquiladora workers:The case of $\mathrm{N}$ ogales, Sonora, Mexico. Int J Health Serv 1995;25:489-502.

15. Moure-Eraso R,W ilcox M, Punnett L, MacD onald L, Levershein C. Back to the future: Sweatshop conditions on the Mexico-U.S. border. II. O ccupational health impact of maquiladora industrial activity. Am J Ind Med 1997;31:587-599.

16.Teagerden M, Butler M,Von Glinow M. Mexico's maquiladora industry: $W$ here strategic human resources management makes a difference. $0 \mathrm{r}$ ganizational Dynamics 1992;1:34-47. 